Bulletin of the Transilvania University of Braşov

Series III: Mathematics and Computer Science, Vol. 1(63), No. 2 - 2021, 29-36

https://doi.org/10.31926/but.mif.2021.1.63.2.3

\title{
ON SOME THEOREMS OF THE JACOBI-LIPSCHITZ CLASS FOR THE JACOBI TRANSFORM
}

\author{
Radouan DAHER, ${ }^{1}$ Nisrine DJELLAB ${ }^{2}$ and Mohamed EL HAMMA*,3
}

\begin{abstract}
Using a generalized Jacobi translation, we obtain a generalization of the theorem 84 of Titchmarsh for the Jacobi transform satisfying the JacobiLipschitz and Dini Lipschitz conditions in the space $\mathrm{L}^{p}\left(\mathbb{R}^{+}, \Delta(t) d t\right)$, where $1<p \leq 2$.
\end{abstract}

2000 Mathematics Subject Classification: 33C45

Key words: Jacobi operator, Generalized Jacobi translation, LipschitzJacobi class, Dini Lipschitz-Jacobi class.

\section{Introduction and preliminaries}

Titchmarsh established in ([15], Theorem 84) that if $f(x)$ satisfies the Lipschitz condition $\operatorname{Lip}(\alpha, p)$ in the $\mathrm{L}^{p}, 1<p \leq 2$, on the real line $\mathbb{R}$, that is

$$
\int_{-\infty}^{+\infty}|f(x+h)-f(x-h)|^{p} d x=O\left(h^{\alpha p}\right), 0<\alpha \leq 1, h \rightarrow 0,
$$

then its Fourier transform $\widehat{f}$ belongs to $\mathrm{L}^{\beta}$ for

$$
\frac{p}{p+\alpha p-1}<\beta \leq \frac{p}{p-1} .
$$

On the other hand, Younis proved this theorem to higher differences and to functions on $\mathbb{R}$ (see [16])) satisfying the Dini-Lipschitz condition. More precisely, we have

\footnotetext{
${ }^{1}$ Laboratoire Mathématiques Fondamentales et Appliqués, Faculté des Sciences Aïn Chock, Université Hassan II, B.P 5366 Maarif, Casablanca, Maroc, e-mail: rjdaher024@gmail.com

${ }^{2}$ Laboratoire Mathématiques Fondamentales et Appliqués, Faculté des Sciences Aïn Chock, Université Hassan II, B.P 5366 Maarif, Casablanca, Maroc, e-mail: nisrine.djellab@gmail.com

3* Corresponding author, Laboratoire Mathématiques Fondamentales et Appliqués, Faculté des Sciences Aïn Chock, Université Hassan II, B.P 5366 Maarif, Casablanca, Maroc, e-mail: m_elhamma@yahoo.fr
} 
Theorem 1. [16] Let $f \in \mathrm{L}^{p}(\mathbb{R})$ with $1<p \leq 2$ such that

$$
\left(\int_{-\infty}^{+\infty}|f(x+h)-f(x)|^{p} d x\right)^{1 / p}=O\left(\frac{h^{\alpha}}{\left(\log \frac{1}{h}\right)^{\gamma}}\right), h \rightarrow 0,0<\alpha \leq 1, \quad \gamma>0
$$

Then, its Fourier transform $\widehat{f}$ belongs to $\mathrm{L}^{\beta}$ for

$$
\frac{p}{p+\alpha p-1}<\beta \leq \frac{p}{p-1} .
$$

The main aim of this paper is to establish an analogue of these theorems for the Jacobi transform setting by means of the generalized Jacobi translation. There are many analogues of these theorems: for the Bessel transform on $\mathbb{R}^{+}$, for the Dunkl transform on $\mathbb{R}^{d}$, for the Laguerre Hypergroup and etc (for exemple, see $[7,9,12])$

Now, we recall some notations and results about harmonic analysis on Jacobi transform and we refer for more details to the articles $[1,8,11,13,14]$

Throughout the paper $\alpha, \beta$ and $\rho$ are arbitrary real numbers with $\alpha>\beta \geq-\frac{1}{2}$ and $\rho=\alpha+\beta+1$

We consider the Jacobi differential operator

$$
\mathrm{D}=\mathrm{D}_{\alpha, \beta}=\frac{d^{2}}{d t^{2}}+((2 \alpha+1) \operatorname{coth} t+(2 \beta+1) \tanh t) \frac{d}{d t} .
$$

It is known that for any $\lambda \in \mathbb{C}$ there exists a unique even $\mathrm{C}^{\infty}$-solution $u(t)$ of the differential equation

$$
\left\{\begin{array}{l}
\mathrm{D} u+\left(\lambda^{2}+\rho^{2}\right) u=0 \\
u(0)=1, \frac{d}{d x} u(0)=0 .
\end{array}\right.
$$

This function $u(t)$ is called Jacobi function and it is denoted $\phi_{\lambda}(t)=\phi_{\lambda}^{(\alpha, \beta)}(t)$. The function $\phi_{\lambda}(t)$ can be expressed in terms of the hypergeometric function

$$
\phi_{\lambda}(t)={ }_{2} F_{1}\left(\frac{1}{2}(\rho-i \lambda), \frac{1}{2}(\rho+i \lambda), \alpha+1,-\sinh ^{2} t\right) .
$$

where ${ }_{2} F_{1}(a, b, c, z)$ is the hypergeometric function. by

For $\alpha \geq-\frac{1}{2}$, we introduce the normalized spherical Bessel function $j_{\alpha}$ defined

$$
j_{\alpha}(z)=\Gamma(\alpha+1) \sum_{k=0}^{\infty} \frac{(-1)^{k}}{k ! \Gamma(k+\alpha+1)}\left(\frac{z}{2}\right)^{2 k}, z \in \mathbb{C},
$$

where $\Gamma(x)$ is the gamma-function.

Moreover, from (1) we see that

$$
\lim _{x \rightarrow 0} \frac{j_{\alpha}(x)-1}{x^{2}} \neq 0 .
$$


by consequence, there exist $c>0$ and $\eta>0$ satisfying

$$
|x| \leq \eta \Longrightarrow\left|j_{\alpha}(x)-1\right| \geq c|x|^{2}
$$

Lemma 1. Let $\alpha>-1 / 2, \alpha>\beta \geq-1 / 2$, and let $t_{0}>0$. Then for $|\eta| \leq \rho$, there exists a positive constant $C_{1}$ such that

$$
\left|1-\phi_{\mu+i \eta}(t)\right| \geq C_{1}\left|1-j_{\alpha}(\mu t)\right|
$$

for all $0 \leq t \leq t_{0}$

Proof. (see Lemma 9 in [4]).

We adhere to the conventions and normalization used [10], the $c$-function

$$
c(\lambda)=\frac{2^{\rho} \Gamma(i \lambda) \Gamma\left(\frac{1}{2}(1+i \lambda)\right)}{\Gamma\left(\frac{1}{2}(i \lambda+\rho)\right) \Gamma\left(\frac{1}{2}(i \lambda+\rho)-\beta\right)} .
$$

The function $|c(\lambda)|^{-2}$ is an even continuous function on $\mathbb{R}$ and satisfies the following estimates. There exist positive constants $k_{1}, k_{2}, k$ such that

1. If $\rho \geq 0$ and $\alpha>-\frac{1}{2}$, then

$$
k_{1}|\lambda|^{2 \alpha+1} \leq|c(\lambda)|^{-2} \leq k_{2}|\lambda|^{2 \alpha+1}, \lambda \in \mathbb{R},|\lambda|>k
$$

2. If $\rho>0$ and $\alpha>-\frac{1}{2}$, then

$$
k_{1}|\lambda|^{2} \leq|c(\lambda)|^{-2} \leq k_{2}|\lambda|^{2}, \lambda \in \mathbb{R},|\lambda| \leq k .
$$

For more details, see $[2,3]$ and references therein.

We denote $\mathcal{D}\left(\mathbb{R}^{+}\right)$is the space of space of even $\mathrm{C}^{\infty}$-function on $\mathbb{R}^{+}$with compact support.

Definition 1. [14] For every $f \in \mathcal{D}\left(\mathbb{R}^{+}\right)$the Jacobi transform of $f$ is defined by:

$$
\mathcal{F}(f)(\lambda)=\widehat{f}(\lambda)=\int_{0}^{\infty} f(t) \phi_{\lambda}(t) \Delta(t) d t, \lambda \in \mathbb{R}^{+},
$$

where $\Delta(t)=(2 \sinh t)^{2 \alpha+1}(2 \cosh t)^{2 \beta+1}$.

The mapping $\mathcal{F}: f \rightarrow \widehat{f}$ extended by continuity from the Banach space $\mathrm{L}^{p}\left(\mathbb{R}^{+}, \Delta(t) d t\right)$ onto the Banach space $\mathrm{L}^{q}\left(\mathbb{R}^{+}, d \mu(\lambda)\right)$, where $d \mu(\lambda)=\frac{1}{\sqrt{2 \pi}}|c(\lambda)|^{-2} d \lambda$ and $p^{-1}+q^{-1}=1$. The extended mapping is also denoted by $\mathcal{F}: f \rightarrow \widehat{f}$ and it is called Jacobi transform.

We have the inversion formula (cf. [11])

$$
f(t)=\int_{0}^{\infty} \widehat{f}(\lambda) \phi_{\lambda}(t) d \mu(\lambda)
$$


We put $\|f\|_{\rho, p}=\|f\|_{L^{p}\left(\mathbb{R}^{+}, \Delta(t) d t\right)}$ and $\|\widehat{f}\|_{\mu, q}=\|\widehat{f}\|_{L^{q}\left(\mathbb{R}^{+}, d \mu(\lambda)\right)}$.

From [5], we have the Hausdorff-Young inequality

$$
\|\widehat{f}\|_{\mu, q} \leq C_{2}\|f\|_{\rho, p}
$$

where $C_{2}>0$ is a positive constant.

The generalized Jacobi translation was defined by Flensted-Jensen and Koornwinder [10] (analogue of translation operator for Fourier transform) given by

$$
\mathrm{T}_{y} f(x)=\int_{0}^{\infty} f(z) K(x, y, z) \Delta(z) d z
$$

with kernel

$$
\begin{aligned}
K(x, y, z) & =\frac{2^{-2 \rho} \Gamma(\alpha+1)(\cosh x \cosh y \cosh z)^{-\alpha-\beta-1}}{\Gamma\left(\frac{1}{2}\right) \Gamma\left(\alpha+\frac{1}{2}\right)(\sinh x \sinh y \sinh z)^{2 \alpha}}\left(1-B^{2}\right)^{\alpha-\frac{1}{2}} \\
& \times{ }_{2} F_{1}\left(\left(\alpha+\beta, \alpha-\beta, \alpha+\frac{1}{2}, \frac{1}{2}(1-B)\right),\right.
\end{aligned}
$$

for $|x-y|<z<x+y$ and $K(x, y, z)=0$ elsewhere and

$$
B=\frac{\cosh ^{2} x+\cosh ^{2} y+\cosh ^{2} z-1}{2 \cosh x \cosh y \cosh z} .
$$

In [4], we have

$$
\widehat{\left(\mathrm{T}_{h} f\right)}(\lambda)=\phi_{\lambda}(h) \widehat{f}(\lambda)
$$

\section{Main results}

In this section, we give the main results of the paper but first we need to define the Lipschitz-Jacobi class.

Definition 2. Let $0<\delta \leq 1$. A function $f(x) \in \mathrm{L}^{p}\left(\mathbb{R}^{+}, \Delta(t) d t\right)$ is said to be in the Lipschtz-Jacobi class, denoted by Lip $(\delta, p)$, if it satisfies

$$
\left\|\mathrm{T}_{h} f(x)-f(x)\right\|_{\rho, p}=O\left(h^{\delta+2}\right) \text {, as } h \rightarrow 0 .
$$

for all $x$ in $\mathbb{R}^{+}$.

Theorem 2. Let $f$ belongs to the Lipschitz-Jacobi class Lip $(\delta, p), \quad 0<\delta \leq 1$ and $1<p \leq 2$. Then $\widehat{f} \in \mathrm{L}^{\gamma}\left(\mathbb{R}^{+}, d \mu(\lambda)\right)$ for all $\gamma$ satisfying

$$
\frac{3 p}{3 p+\delta p-3}<\gamma \leq \frac{p}{p-1}
$$


Proof. Let $f \in \mathrm{L}^{p}\left(\mathbb{R}^{+}, \Delta(t) d t\right)$ satisfying relation (6)

i.e.,

$$
\left\|\mathrm{T}_{h} f(x)-f(x)\right\|_{\rho, p}=O\left(h^{\delta+2}\right), \text { as } h \rightarrow 0 .
$$

Thus, from formula (5) and the Hausdorff-Young inequality, we obtain

$$
\int_{0}^{+\infty}\left|1-\phi_{\lambda}(h)\right|^{q}|\widehat{f}(\lambda)|^{q} d \mu(\lambda) \leq C_{2}^{q}\left\|\mathrm{~T}_{h} f(x)-f(x)\right\|_{\rho, p}^{q}
$$

Then

$$
\int_{0}^{+\infty}\left|1-\phi_{\lambda}(h)\right|^{q}|\widehat{f}(\lambda)|^{q} d \mu(\lambda) \leq K h^{q(\delta+2)},
$$

where $K>0$ is a positive constant.

Lemma 1 implies that

$$
C_{1} \int_{0}^{+\infty}\left|1-j_{\alpha}(\lambda h)\right|^{q}|\widehat{f}(\lambda)|^{q} d \mu(\lambda) \leq K h^{q(\delta+2)}
$$

i.e.,

$$
\int_{0}^{+\infty}\left|1-j_{\alpha}(\lambda h)\right|^{q}|\widehat{f}(\lambda)|^{q} d \mu(\lambda) \leq C_{3} h^{q(\delta+2)}
$$

where $C_{3}=\frac{K}{C_{1}}>0$ is a positive constant.

It follows from (2) that

$$
c^{q} \int_{0}^{\frac{\eta}{h}}|\lambda h|^{2 q}|\widehat{f}(\lambda)|^{q} d \mu(\lambda) \leq C_{3} h^{q(\delta+2)}
$$

Therefore

$$
\int_{0}^{\frac{\eta}{h}}\left|\lambda^{2} \widehat{f}(\lambda)\right|^{q} d \mu(\lambda) \leq C_{4} h^{q \delta}
$$

where $C_{4}=C_{3} c^{-q}$.

Now, let

$$
\psi(t)=\int_{1}^{t}\left|\lambda^{2} \widehat{f}(\lambda)\right|^{\gamma} d \mu(\lambda)
$$

Then, for $\gamma \leq q$, where $\frac{1}{p}+\frac{1}{q}=1$, by Hölder inequality, we have

$$
\begin{aligned}
\psi(t) & \leq\left(\int_{1}^{t}\left|\lambda^{2} \widehat{f}(\lambda)\right|^{q} d \mu(\lambda)\right)^{\frac{\gamma}{q}}\left(\int_{1}^{t} d \mu(\lambda)\right)^{1-\frac{\gamma}{q}} \\
& \leq\left(\int_{1}^{t}\left|\lambda^{2} \widehat{f}(\lambda)\right|^{q} d \mu(\lambda)\right)^{\frac{\gamma}{q}}\left(\int_{1}^{t}|c(\lambda)|^{-2} d \lambda\right)^{1-\frac{\gamma}{q}}
\end{aligned}
$$


whence, in view of (4) and (7), we obtain

$$
\psi(t) \leq O\left(t^{-\delta \gamma}\right) t^{3-3 \frac{\gamma}{q}}
$$

That is

$$
\int_{1}^{t}\left|\lambda^{2} \widehat{f}(\lambda)\right|^{\gamma} d \mu(\lambda)=O\left(t^{-\delta \gamma+3-3 \frac{\gamma}{q}}\right)=O\left(t^{-\delta \gamma+3-3 \gamma+\frac{3 \gamma}{p}}\right)
$$

Since $\lambda \geq 1$, then

$$
\begin{aligned}
\int_{1}^{t}|\widehat{f}(\lambda)|^{\gamma} d \mu(\lambda) & \leq \int_{1}^{t}\left|\lambda^{2} \widehat{f}(\lambda)\right|^{\gamma} d \mu(\lambda) \\
& =O\left(t^{-\delta \gamma+3-3 \gamma+\frac{3 \gamma}{p}}\right)
\end{aligned}
$$

and this is bounded as $t \rightarrow \infty$ if

$$
-\delta \gamma+3-3 \gamma+\frac{3 \gamma}{p}<0
$$

which gives

$$
\frac{3 p}{3 p+p \delta-3}<\gamma \leq \frac{p}{p-1}
$$

and this ends the proof.

By analog with the proof of Theorem 2, we can establish the following result

Theorem 3. If $f \in \mathrm{L}^{p}\left(\mathbb{R}^{+}, \Delta(t) d t\right)$ with $1<p \leq 2$ such that

$$
\left\|\mathrm{T}_{h} f(x)-f(x)\right\|_{\rho, p}=O\left(\frac{h^{\delta+2}}{\left(\log \frac{1}{h}\right)^{\sigma}}\right), \text { as } h \rightarrow 0,0<\delta \leq 1, \sigma>0
$$

Then $\widehat{f} \in \mathrm{L}^{\gamma}\left(\mathbb{R}^{+}, d \mu(\lambda)\right)$ for all $\gamma$ satisfying

$$
\frac{3 p}{3 p+p \delta-3}<\gamma \leq \frac{p}{p-1} .
$$

\section{Acknowledgments}

The authors would like to thank the referees for their valuable comments and suggestions. 
On some theorems of the Jacobi-Lipschitz...

\section{References}

[1] Anker, J.P, Damek, E. and Yacoub, C., Spherical analysis on harmonic AN group, Ann. Scuola. Norm. Sup. Pisa 23 (1996), 643-679.

[2] Bloom, W.R and Xu, Z., Local Hardy spaces on Chèli-Trimèche hypergroups, Stud. Math. 133 (1999), no. 3, 197-230.

[3] Bloom, W.R. and Xu, Z., The Hardy-Littlewood maximal function for ChèliTrimèche hypergroups, Contemp. Math. 183 (1995), 45-69.

[4] Bray, W.O. and Pinsky, M.A., Growth properties of Fourier transforms via moduli of continuity, Journal of Functional Analysis 255 (2008), 2265-2285.

[5] Chokri, A and Jemai, A., Integrability theorems for Fourier-Jacobi transform, J. Math. Inequal. 6 (2012), no. 3, 343-353.

[6] Daher, R. and El Hamma, M., An analog of Titchmarsh's theorem of Jacobi transform, Int. J. Math. Analy, 6, (2012), no. 20, 975-981.

[7] Daher, R., El Hamma, M. and Akhlidj, A., Dini-Lipschitz functions for the Bessel transform, Nonlinear studies 24 (2017), no. 2, 297-301.

[8] El Hamma, M. and Daher, R., Equivalence of $K$-functionals and modulus of smoothness constructed by generalized Jacobi transform, Integral Transforms and Special Functions 30 (2019), no. 12, 1018-1024. doi:10.1080/10652469.2019. 1635127.

[9] El Hamma, M. and Daher, R., On some theorems of the Dunkl-Lipschitz class for the Dunkl transform, Lobachevskii Journal of Mathematics 40 (2019), no. 8, 1157-1163.

[10] Flensted-Jensen, M. and Koornwinder, T., The convolution structure for Jacobi function expansions, Ark. Mat. 11 (1973), 245-262.

[11] Koornwinder, T.H., Jacobi functions and analysis on noncompact semisimple Lie group, Group Theoretical Aspects and Applications, Askey R. et al (eds.), D. Reidel Publishing Company Dordrecht, 1984, 1-85.

[12] Negzaoui, S., Lipschitz conditions in Lagurre Hypergroup, Mediterr. J. Math (2017) 14:191 Doi 10.1007/s00009-017-0989-4.

[13] Platonov, S.S., Fourier-Jacobi harmonic analysis and some problems of approximation of functions on the half-axis in $\mathrm{L}_{2}$ metric: Nikol'skiiBesov type functions spaces, Integral Transforms and Special Functions, DOI:10.1080/10652469.2019.1691548.

[14] Platonov, S.S., Fourier-Jacobi harmonic analysis and some problems of approximation of functions on the half-axis in $\mathrm{L}_{2}$ metric: Jackson's type direct theorems, Integral Transforms and Special Functions, DOI:10.1080/10652469.2018.1562449. 
[15] Titchmarsh, E. C., Introduction to the theory of Fourier integrals, Clarendon Press, Oxford, 1948.

[16] Younis, M.S., Fourier transforms of Dini-Lipschitz functions, J. Math. Math. Sci. 9 (1986), no. 2, 301-312. 